\title{
Effect of ensiled mulberry leaves and sun-dried mulberry fruit pomace on the fecal bacterial community composition in finishing steers
}

Yan Li ${ }^{1,2}$, Qingxiang Meng ${ }^{1,2}$, Bo Zhou ${ }^{1,2}$ and Zhenming Zhou ${ }^{1,2^{*}}$

\begin{abstract}
Background: Here, we aimed to investigate the effects of ensiled mulberry leaves (EML) and sun-dried mulberry fruit pomace (SMFP) on fecal bacterial communities in Simmental crossbred finishing steers. To this end, the steers were reared on a standard TMR diet, standard diet containing EML, and standard diet containing SMFP. The protein and energy levels of all the diets were similar. Illumina MiSeq sequencing of the V4 region of the $16 \mathrm{~S}$ rRNA gene and quantitative real-time PCR were used to analyze and detect the fecal bacterial community.

Results: Most of the sequences were assigned to Firmicutes (56.67\%) and Bacteroidetes (35.90\%), followed by Proteobacteria (1.87\%), Verrucomicrobia (1.80\%) and Tenericutes (1.37\%). The predominant genera were 5-7 N15 (5.91\%), CF231 (2.49\%), Oscillospira (2.33\%), Paludibacter (1.23\%) and Akkermansia (1.11\%). No significant differences were observed in the numbers of Firmicutes ( $p=0.28)$, Bacteroidetes $(p=0.63)$, Proteobacteria $(p=0.46)$, Verrucomicrobia $(p=0.17)$, and Tenericutes ( $p=0.75)$ populations between the treatment groups. At the genus level, genera classified with high abundance (more than $0.1 \%$ ) belonged primarily to Bacteroidetes and Firmicutes. Furthermore, no differences were observed at the genus level: 5-7 N15, CF231, Oscillospira, Paludibacter, and Akkermansia ( $p>0.05$ in all cases), except that $r(4-4$ was lower in the CON and SMFP groups than in the EML group $(p=0.02)$. There were no significant differences in the richness estimate and diversity indices between the groups $(p>0.16)$, and the different diets did not significantly influence most selected fecal bacterial species $(p>0.06$ ), except for Ruminococcus albus, which was higher in the EML group $(p<0.01)$ and Streptococcus bovis, which was lower in the CON group $(p<0.01)$ relative to the other groups.
\end{abstract}

Conclusions: In conclusion, diets supplemented with EML and SMFP have little influence on the fecal bacterial community composition in finishing steers.

Keywords: Fecal bacteria community composition, Sequencing, Ensiled mulberry leaves (EML), Sun-dried mulberry fruit pomace (SMFP)

\section{Background}

With the increased demand for animal production in many developing countries, including China, there is an obvious demand for sufficient and inexpensive livestock feed. Several reports have shown that agricultural byproducts and fruit residue can be used to replace cerealbased concentrates as livestock feed without negatively

\footnotetext{
* Correspondence: zhouzm@cau.edu.cn

${ }^{1}$ State Key Laboratory of Animal Nutrition, Beijing 100193, People's Republic of China

${ }^{2}$ College of Animal Science and Technology, China Agricultural University, Beijing 100193, People's Republic of China
}

affecting animal production performance. Previous studies have compared the dry matter digestibility of several grape pomace varieties in cows, sheep, and goats in vitro [1-3], and in vivo experiments have investigated feeds such as apple pomace silage [4], which is used for sheep [5], and potato pomace, which is used as protein supplement for growing lambs [6]. Mulberry pomace, which is a byproduct of mulberry juice, consists mainly of peels and stems and accounts for approximately $8 \%$ of the fresh weight of the mulberry [7-10]. In our previous study, we found that ensiled mulberry leaves (EML) 
contains $19.8 \%$ crude protein (CP), $15.6 \%$ water soluble carbohydrates (WSC), and 51.5\% neutral detergent fiber (NDF), and sun-dried mulberry fruit pomace (SMFP) contains $21.8 \% \mathrm{CP}, 20.8 \% \mathrm{WSC}$, and $49.1 \%$ NDF [11]. Furthermore, both EML and SMFP can be used in finishing steers diets without impairing their productive performance and carcass characteristics $[12,13]$.

However, little is known about how EML and SMFP could influence the fecal microbial community structure. The intestinal microbiome of cattle plays a critical role not only in animal health and productivity but also in food safety and environmental protection [14]. A previous survey suggested that bovine fecal bacterial community structures can dramatically differ at the phylum and family levels depending on the animal feed. Furthermore, the feeding operation has been reported to be a more important determinant of the cattle microbiome than the geographic location of the feedlot $[15,16]$. Our hypothesis is that EML and SMFP can be used in the diets for finishing steers without affecting the fecal bacterial community composition, and harmless to animal health and food safety. To validate this assumption, we used sequencing analysis to examine the effects of EML and SMFP on the fecal bacterial communities in cattle fed these byproducts.

\section{Methods}

\section{Animals and diets}

The animals in this study were handled in strict accordance with the Regulations for Laboratory Animals of Beijing. The protocol was approved by the Animal Welfare Committee of China Agricultural University (Permit No.DK1008). Experiments were performed in accordance with the Regulations for the Administration of Affairs Concerning Experimental Animals (The State Science and Technology Commission of P. R. China, 1988).

This study was part of a larger experimental trial investigating the effects of EML and SMFP on growth performance, ruminal fermentation, blood biochemical parameters, and carcass characteristics of finishing steers [11-13]. The experiment was done in the experimental base of China Agricultural University. A total of 51 healthy Simmental crossbred steers weighing $357.06 \pm 16.5 \mathrm{~kg}$ (average age, 15 months) were divided into three treatment groups $(n=17)$ and each group was fed a different diet. The control group $(\mathrm{CON})$ received standard TMR, the EML received a standard diet containing EML, and the sun-dried mulberry fruit pomace group (SMFP) received a standard diet containing SMFP (Additional file 1: Table S1). The ingredients and nutrient composition of each diet are provided in Additional file 1: Table S1 and in our previous report [11]. The animals were fed twice daily at approximately 0800 and $1700 \mathrm{~h}$ to meet the NRC recommendations and had ad libitum access to water. To investigate the effects of EML and SMFP on the fecal bacterial community, 4 steers were selected randomly in every treatment, and totally 12 steers were selected.

\section{Fecal sampling}

At the end of the experiment, 30-50 g fecal samples were obtained aseptically from cattle rectums with a new palpation sleeve used for each sample. The fecal samples were quickly sealed in $50-\mathrm{mL}$ conical tubes. Sampling was accomplished as quickly as possible. The samples were then frozen in liquid nitrogen prior to storage at $-80^{\circ} \mathrm{C}$ until genomic DNA was extracted.

\section{DNA extraction and sequencing}

Total genomic DNA was extracted from $0.2 \mathrm{~g}$ of frozen fecal sample using a fecal DNA extraction toolkit (Tiangen Biotech Co., Beijing, China) combining a beadbeat with an oscillator (Precellys 24, Bertin Technology, Montigny-le-Bretonneux, France) plus column method [17]. The rotating speed of the oscillator was $5500 \mathrm{rpm}$ with two circulations and $30 \mathrm{~s}$ per circulation. RNA was digested with $50 \mu \mathrm{g}$ RNase A, and DNA was subsequently cleaned up and eluted in $50 \mu \mathrm{L}$ of EB. The DNA samples were quantified using a NanoDrop 2000 spectrophotometer (Thermo Fisher Scientific Inc., Wilmington, DE). Subsequently, DNA samples were diluted in TE buffer to obtain a concentration of $5 \mathrm{mM}$. Sequencing was conducted on an Illumina MiSeq platform v2 $2 \times 250$-bp paired-end protocol yielding paired-end reads. Briefly, DNA was amplified using the universal eubacterial primer set (515f: $5^{\prime}$-GTG CCA GCM GCC GCG GTA A-3', 806r: 5'-XXX XXX GGA CTA CHV CCC TWT CTA AT-3'), which targets the hypervariable V4 region of the $16 S$ rRNA gene, with the reverse primer containing a 6-bp error-correcting barcode unique to each sample [18]. Amplification was performed using Phusion High-Fidelity PCR Mastermix (New England Biolabs [Beijing] Ltd., China). The PCR conditions consisted of $3 \mathrm{~min}$ at $94{ }^{\circ} \mathrm{C}$, followed by $35 \mathrm{cy}$ cles of $15 \mathrm{~s}$ at $94{ }^{\circ} \mathrm{C}, 15 \mathrm{~s}$ at $58{ }^{\circ} \mathrm{C}, 10 \mathrm{~s}$ at $68^{\circ} \mathrm{C}$, and a final elongation step of $30 \mathrm{~s}$ at $68^{\circ} \mathrm{C}$. Amplicons were selected on $2 \%$ agarose gels on E-Gel ${ }^{\circ}$ Size Select ${ }^{\text {tw }}$ Agarose Gel and then purified with Agencourt ${ }^{\circ}$ AMPure $^{\circ}$ XP Reagent. The purified DNA was quantified with Quant-iTTM Technology (Life Technologies, Inc.) applying QuantiTTM dsDNA Broad-Range Assay Kit. An Agilent 2100 Bioanalyzer $^{\mathrm{nax}}$ with a High-Sensitivity DNA Kit (Agilent Technologies, Inc., Santa Clara, CA) was used to analyze library sizes and molar concentrations. Emulsion PCR was performed using the Ion OneTouch ${ }^{\text {tw }} 200$ Template Kit v2 DL (Life Technologies, Inc.) according to the manufacturer's instructions. 


\section{Sequence analysis}

The Illumina MiSeq sequencing data were analyzed using QIIME software (version 1.7.0). Filters were applied to sequences prior to phylogenetic analysis. Depending upon the appropriate fragment size for V4 PCR (150-200 bp), bases after position 200 were trimmed and reads shorter than $150 \mathrm{bp}$ were removed. Reads with a quality score of $<25$ were removed with the NGSQC Toolkit, and only sequences without any ambiguous characters were included in the analysis. To calculate the downstream diversity (alpha and beta diversity), all the samples were subsampled to an equal size of 100,000 reads before comparison of the bacterial communities. The sequences were clustered into operational taxonomic units (OTUs) at the $97 \%$ sequence identity level, and the most abundant sequence of each OTU was chosen as a representative. Based on the OTUs, rarefaction curve and alpha diversity indices (i.e., abundance-based coverage estimator [ACE], and the Chao1, Shannon, and Simpson estimators) were developed. The jackknifed beta diversity included the calculated unweighted and weighted Unifrac distances, which were visualized by principal coordinate analysis ( $\mathrm{PCoA}$ ) [19]. A two-dimensional hierarchical clustering heatmap was drawn based on the number of reads of each OTU using R software (version 3.2.4).

\section{Quantitative real-time PCR}

Quantitative real-time PCR with specific primers and probes was used to determine the population size of thirteen major fecal bacteria. All the $\mathrm{qPCR}$ assays were quantified using SYBR Green PCR RealMaster Mix (Tiangen Biotech, co., LTD, China) on an ABI-7300 Prism real-time PCR instrument (ABI, Foster City, CA). The following PCR program was used: one cycle of $95{ }^{\circ} \mathrm{C}$ for $15 \mathrm{~min}$ (initial denaturation), 40 cycles of $95{ }^{\circ} \mathrm{C}$ for $15 \mathrm{~s}$ (denaturation) and $60{ }^{\circ} \mathrm{C}$ for $32 \mathrm{~s}$ (annealing). Following qPCR, the products of amplification were confirmed by agarose gel (1.2\%) electrophoresis. To minimize variations, all real-time PCR assays were performed in triplicate. The abundance of fecal microbes was recorded and multiplied by the dilution factor to determine the total number of target microbes per gram (wet weight).

\section{Statistical analysis}

The read number, sample coverage, unique OTUs, sample richness (Chaol and ACE), and sample diversity (Shannon-Wiener and Simpson's indices) were compared with the general linear model (GLM) and one-way analysis of variance (ANOVA) using SAS 9.0 (SAS Institute, Cary, NC). Abundance of phylum and genus was determined to assess the effects of EML and SMFP supplementation. Absolute abundance of microbes was expressed as copies of $16 \mathrm{~S}$ rRNA genes per gram (wet weight). Statistical significance was set at $p \leq 0.05$.

\section{Results}

\section{Sequencing and general bacterial community composition}

A total of 2,245,345 quality-checked sequences were obtained from the 12 samples, and 133,780-249,943 sequences were returned for each sample. After OTU picking and chimera checking, a total of 17,761 OTUs were calculated for the 12 samples at 3\% dissimilarity. The average OTUs for the CON, EML, and SMFP group were 7498,6933 , and 6503 per sample, respectively. After normalization to 100,000 reads, richness estimates and diversity indices were developed (Table 1). Good's coverage for each sample ranged from 0.9679 to 0.9853 , with a mean value of 0.9748 for all samples. The rarefaction curve (Additional file 1: Figure S1) indicated that a reasonable number of individual samples had been obtained. The most abundant phyla for all 12 samples were Firmicutes (56.67\%), Bacteroidetes (35.90\%), Proteobacteria (1.87\%), Verrucomicrobia (1.80\%), and Tenericutes (1.37\%; Table 2). The minor phyla, accounting for less than $1 \%$ of the bacterial communities was Lentisphaerae $(0.43 \%)$, and the other known phyla account for $1.96 \%$ of the total sequences. Figure 1 shows the average relative abundance of bacterial phyla in the fecal samples. Figure 2 illustrates the community composition of individual

Table 1 Dietary treatments for unique OTUs, richness estimates, and diversity indices within the fecal content

\begin{tabular}{llllll}
\hline \multirow{2}{*}{ Item } & \multicolumn{3}{l}{ Treatment $^{\mathrm{a}}$} & \multicolumn{3}{c}{ SEM $^{\mathrm{b}}$} & $p$ value \\
\cline { 2 - 4 } & CON & EML & SMFP & & \\
\cline { 1 - 4 } SeqsNum & $166,882.30$ & $184,494.30$ & $178,779.30$ & $19,052.18$ & 0.8 \\
OTUsNum & 7498.00 & 6932.75 & 6503.25 & 610.56 & 0.54 \\
EvenSeqsNum & 100,000 & 100,000 & 100,000 & - & - \\
EvenOTUsNum & 5866.00 & 5253.00 & 5012.75 & 346.74 & 0.25 \\
PD-whole-tree & 214.29 & 196.79 & 191.16 & 10.17 & 0.29 \\
Observed & 5866.00 & 5253.00 & 5012.75 & 346.74 & 0.25 \\
species & & & & & \\
Good's & 0.97 & 0.98 & 0.98 & 0.00 & 0.22 \\
coverage & & & & & \\
Richness & & & & & \\
estimate & & & & & \\
Chao1 & $11,386.85$ & 9787.32 & 8929.23 & 834.02 & 0.16 \\
ACE & $11,616.52$ & 9930.3 & 8982.59 & 937.71 & 0.19 \\
Diversity indices & & & & & \\
Simpson & 1.00 & 1.00 & 1.00 & 0.00 & 0.45 \\
Shannon & 9.70 & 9.58 & 9.50 & 0.10 & 0.37 \\
\hline
\end{tabular}

${ }^{\mathrm{a} C O N}$ Control $(n=4)$, EML Ensiled mulberry leaves $(n=4)$, SMFP Sun-dried mulberry fruit pomace $(n=4)$

${ }^{\mathrm{b}} \mathrm{SEM}$ : standard error of the mean 
Table 2 Effect of EML and SMFP diet on changes in phyla (as a percentage of the total number of sequences) in the fecal bacterial community

\begin{tabular}{|c|c|c|c|c|c|}
\hline \multirow[t]{2}{*}{ Phylum } & \multicolumn{3}{|c|}{ Experimental diet ${ }^{a}$} & \multirow[t]{2}{*}{$\mathrm{SEM}^{\mathrm{b}}$} & \multirow[t]{2}{*}{$p$ value } \\
\hline & $\mathrm{CON}$ & EML & SMFP & & \\
\hline Firmicutes & 56.79 & 58.06 & 55.18 & 1.39 & 0.28 \\
\hline Bacteroidetes & 35.09 & 35.34 & 37.28 & 1.6 & 0.63 \\
\hline Proteobacteria & 2.16 & 1.62 & 1.83 & 0.28 & 0.46 \\
\hline Verrucomicrobia & 1.99 & 1.47 & 1.94 & 0.18 & 0.17 \\
\hline Tenericutes & 1.27 & 1.41 & 1.42 & 0.15 & 0.75 \\
\hline Lentisphaerae & 0.63 & 0.32 & 0.32 & 0.18 & 0.41 \\
\hline Others & 2.06 & 1.78 & 2.03 & 0.18 & 0.59 \\
\hline Firmicutes:Bacteroidetes & 1.61 & 1.64 & 1.48 & 0.12 & 0.54 \\
\hline
\end{tabular}

${ }^{a}$ CON Control $(n=4)$, EML Ensiled mulberry leaves $(n=4)$, SMFP Sun-dried mulberry fruit pomace $(n=4)$

${ }^{\mathrm{b}}$ SEM Standard error of the mean

Different letters in the same row indicate a significant difference between values within the row $(p<0.05)$

samples in the different treatments. The bar chart illustrates seven bacterial phyla identified in the fecal samples with high relative abundance. A total of 282 genera were detected from the 12 fecal samples. Known genera with high abundance (more than $0.1 \%$ ) belonged primarily to the phyla Bacteroidetes and Firmicutes, but they all had low abundance, and only five of these genera accounted for $>1 \%$ of total sequences. These five "predominant" genera were 5-7 N15 (5.91\%), CF231 (2.49\%), Oscillospira (2.33\%), Paludibacter (1.23\%), and Akkermansia (1.11\%). Minor genera such as Dorea, rc4-4, Prevotella, Methanobrevibacter, and Campylobacter accounted for 0.98, 0.93, 0.75, 0.57, and $0.19 \%$ of the total sequences, respectively. The other known genera accounting for 4.23 and $79.23 \%$ of the sequences represent strains that were unclassified at the genus level (Table 3).

\section{Changes in the fecal microbiota by the EML and SMFP diets}

Table 1 shows the OTUs, richness estimate, and diversity indices of all the 12 fecal samples across the three groups. The results of the Illumina MiSeq sequencing analyses revealed that addition of EML and SMFP to the feed did not affect the alpha or beta diversity of fecal bacteria (Table 1). The ACE and Chao1 indices were calculated to compare species richness by estimating the minimum number of unique OTUs for each sample. The Chaol $(p=0.16)$ and ACE $(p=0.19)$ values indicated that species richness was the highest in the CON group; however, there was no significant difference in the species richness between groups. Similarly, the Shannon-Wiener $(p=0.37)$ and Simpson $(p=0.45)$ indices both showed that the bacterial community exhibited considerable diversity, but with no differences between groups. The Venn diagram in Fig. 3 illustrates the distribution of all unique, shared, and common phylotypes among $16 S$ rRNA gene libraries prepared from the fecal samples from the three groups. An examination of the phylotypes showed that there were 5468 phylotypes in the fecal communities in all three groups (Fig. 3). Furthermore, steers from the CON group were found to have more phylotypes over the entire course of the experiment $(11,754$ OTU) compared to those from the EML and SMFP groups $(10,521$ and 10,157 OUT, respectively), which was in line with the richness estimate results. A thermal double dendrogram of the 50 most

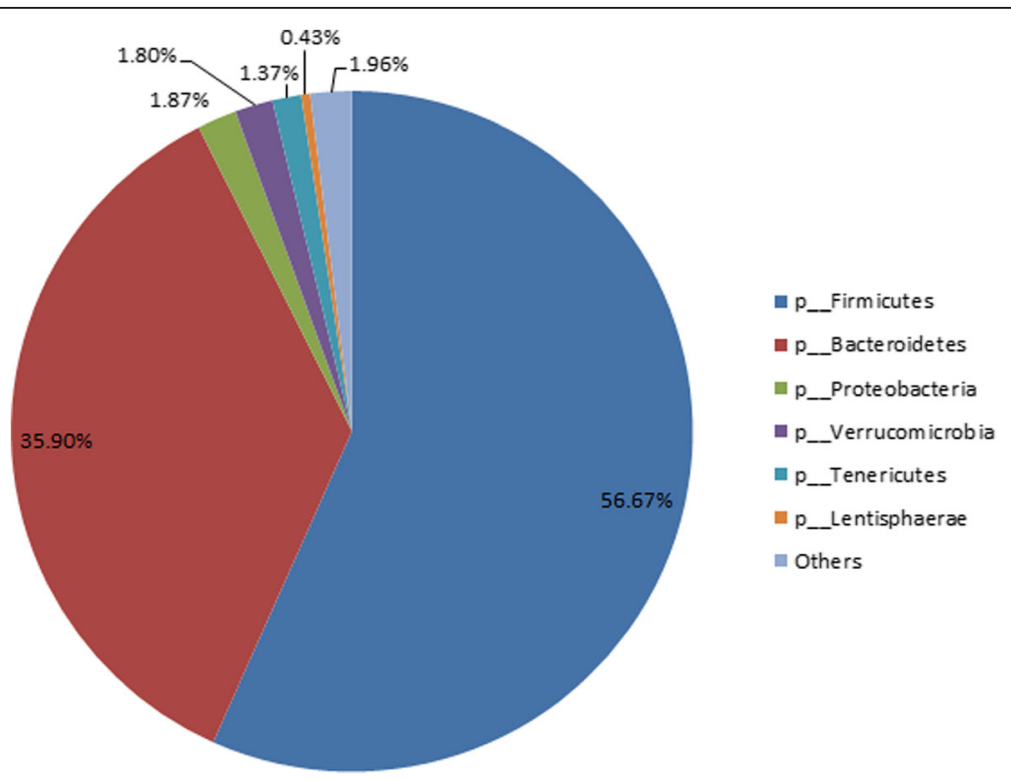

Fig. 1 Average relative abundance of fecal bacteria in steers fed different diets 


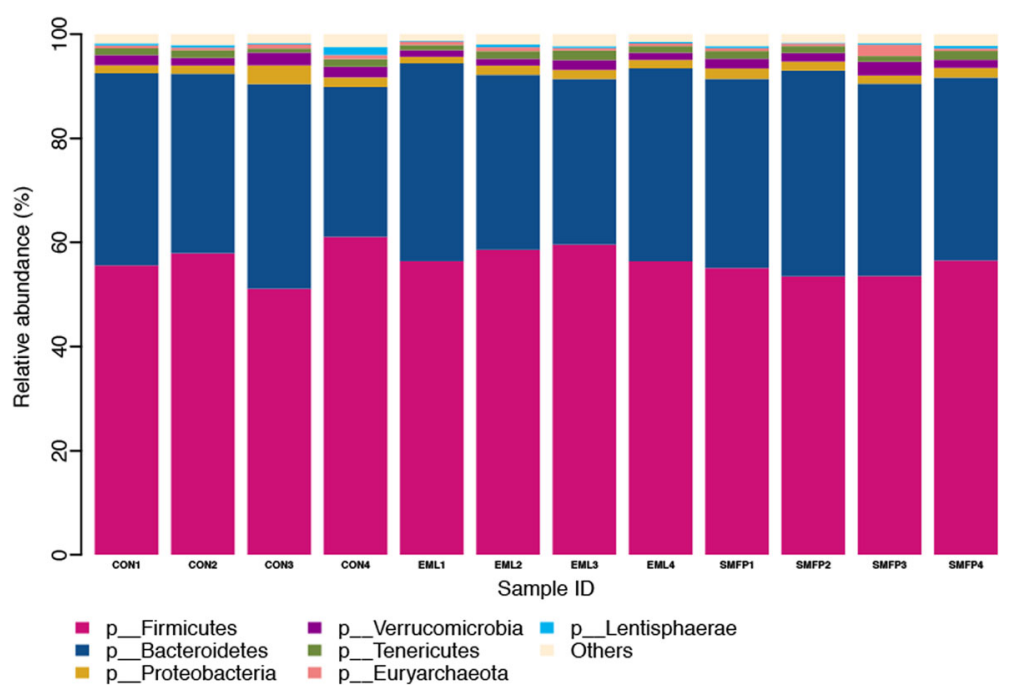

Fig. 2 Bar chart showing the relative abundance of fecal bacterial composition at the phylum level

abundant bacterial OTUs (Additional file 1: Figure S2) demonstrated that samples in the same treatment could not be easily grouped, indicating that there were considerable similarities in the fecal bacterial communities of the different treatment groups.

The distribution was based on a $3 \%$ species cutoff. The $16 \mathrm{~S}$ gene libraries were prepared from fecal samples obtained from cattle fed the CON $(n=4)$, EML $(n=4)$, and $\operatorname{SMFP}(n=4)$ diets.

Table 3 Effect of EML and SMFP on changes in the genus composition (as a percentage of the total number of sequences) in the fecal bacterial community

\begin{tabular}{|c|c|c|c|c|c|}
\hline \multirow[t]{2}{*}{ Genus } & \multicolumn{3}{|c|}{ Experimental diet ${ }^{1}$} & \multirow[t]{2}{*}{$\mathrm{SEM}^{2}$} & \multirow[t]{2}{*}{$p$ value } \\
\hline & $\mathrm{CON}$ & EML & SMFP & & \\
\hline 5-7 N15 & 5.27 & 5.95 & 6.51 & 0.45 & 0.21 \\
\hline CF231 & 2.36 & 2.16 & 2.95 & 0.59 & 0.62 \\
\hline Oscillospira & 2.31 & 2.35 & 2.33 & 0.07 & 0.90 \\
\hline Paludibacter & 1.12 & 1.18 & 1.37 & 0.12 & 0.33 \\
\hline Akkermansia & 1.14 & 0.91 & 1.28 & 0.18 & 0.37 \\
\hline Dorea & 0.96 & 1.03 & 0.95 & 0.08 & 0.78 \\
\hline rc4-4 & $0.88^{\mathrm{b}}$ & $1.21^{\mathrm{a}}$ & $0.71^{\mathrm{b}}$ & 0.10 & 0.01 \\
\hline [Prevotella] & 0.57 & 0.65 & 1.02 & 0.15 & 0.12 \\
\hline Methanobrevibacter & 0.46 & 0.47 & 0.77 & 0.26 & 0.65 \\
\hline Campylobacter & 0.50 & 0.01 & 0.07 & 0.27 & 0.41 \\
\hline Others & 4.02 & 4.29 & 4.55 & 0.23 & 0.30 \\
\hline Unknown & 80.40 & 79.79 & 77.48 & 1.53 & 0.40 \\
\hline
\end{tabular}

${ }^{1}$ CON Control $(n=4)$, EML Ensiled mulberry leaves $(n=4)$, SMFP Sun-dried mulberry fruit pomace $(n=4)$

${ }^{2}$ SEM Standard error of the mean

Different letters in the same row indicate a significant difference between values within the row $(p<0.05)$
To assess the potential effect of EML and SMFP diets on fecal bacterial communities, sequencing datasets from individual groups of steers were examined (Table 2 ). Both diets had no influence on the proportion of Firmicutes, which is a major gram-positive phylum in the fecal samples $(p=0.28)$. Similarly, the proportion of the major gram-negative phylum Bacteroidetes was also unchanged by the EML and SMFP diets $(p=0.63)$. There were no statistically significant differences in the less abundant bacterial phyla among the three groups $(p>0.10$; Table 2). To evaluate the effects of the EML

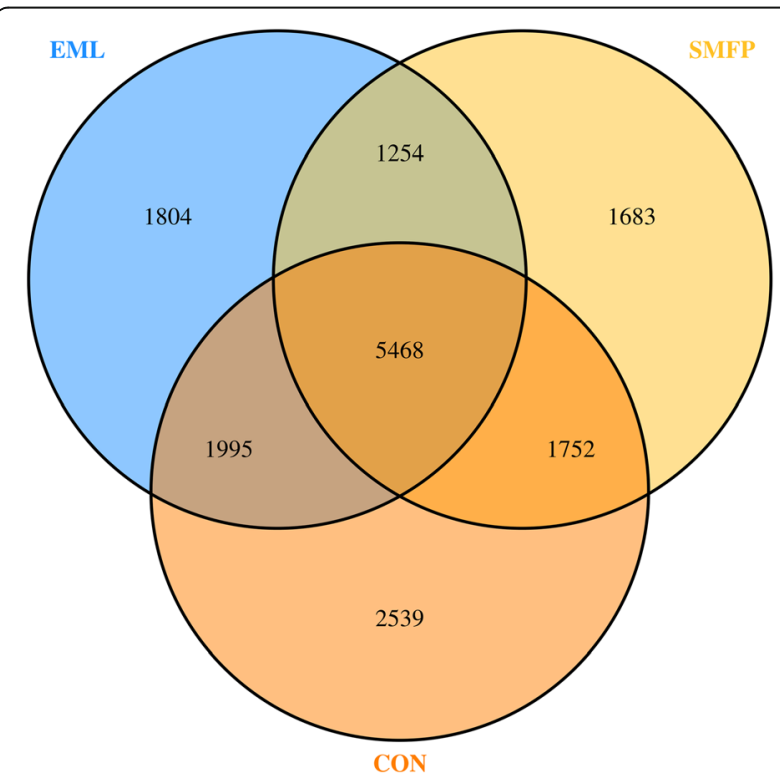

Fig. 3 Venn diagram illustrating the distribution of unique, shared, and common phylotypes among 165 rRNA gene libraries in the three groups 
and SMFP diets on the fecal bacterial community composition, genera with an abundance of $>0.3 \%$ were selected. Most of the selected genera did not show a remarkable difference among the three groups $(p>0.05)$, except for $r c 4-4(p=0.01)$, which was increased in the EML group (Table 3 ). The above data indicated that the bacterial community did not significantly differ between the three groups.

Figure 4 presents a comparison among the 12 samples by PCoA. The weighted and unweighted PCoA indicated that the relative abundances of bacterial populations were not affected by diets All the sample points were equally distributed in the coordinates (weighted and unweighted); meanwhile, the three clusters could not be obviously separated from each other, i.e., the bacterial communities of the three groups did not significantly differ. The difference between the groups was less obvious, and the CON group was not distinguishable from the EML and SMFP groups.

Weighted PCoA was based on the relative abundance of bacterial communities, while unweighted PCoA was based on the uniqueness of the bacterial communities. CON, control group $(n=4)$; EML, ensiled mulberry leaves $(n=4)$; SMFP, sun-dried mulberry fruit pomace $(n=4)$.

\section{Proportion of selected bacteria in feces}

Table 4 presents the results of the real-time qPCR analyses of 13 selected fecal bacteria. As shown in the table, EML and SMFP affected the composition of fecal bacteria, as reflected by the specific species. Compared with the CON group, the populations of Ruminococcus albus $(p=0.0015)$ and Streptococcus bovis $(p=0.0086)$ were
Table 4 Fecal bacterial abundance in finishing steers fed a total mixed ration supplemented with EML and SMFP

\begin{tabular}{|c|c|c|c|c|c|}
\hline \multirow[t]{2}{*}{ Bacteria } & \multicolumn{3}{|c|}{ Experimental diet ${ }^{1}$} & \multirow[t]{2}{*}{ SEM $^{2}$} & \multirow[t]{2}{*}{$P$ value } \\
\hline & $\mathrm{CON}$ & EML & SMFP & & \\
\hline Fibrobacter succinogenes & 0.0268 & 0.0280 & 0.0361 & 0.0149 & 0.8933 \\
\hline ii & 6 & 0.0068 & 07 & 0.0032 & $c$ \\
\hline revotella ruminicola & 0.0005 & 0.0089 & 0.0008 & 0.0049 & 0.4510 \\
\hline mir & 0.1069 & 0.12 & 0 & 15 & 0 \\
\hline elenomonas ruminantium & 0.0725 & 0.1424 & 0.1277 & 0.0170 & 0.0591 \\
\hline vens & 0.0993 & 3.2201 & 3.9093 & 0.9618 & 0.0652 \\
\hline Butyrivibrio fibrisolvens & 0.0001 & 0.0170 & 0.0179 & 0.0049 & 0.0702 \\
\hline ubacterium rumina & 0.0 & 0.00 & 0.0 & 0.0 & $c$ \\
\hline Megasphaera elsdenii & 0.0001 & 0.0105 & 0.0060 & 0.0031 & 0.1337 \\
\hline 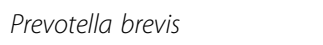 & 0.0000 & 0.0021 & 0.0009 & 0.0007 & 0.1 \\
\hline Ruminobacter amylophilus & 0.0000 & 0.0484 & 0.0038 & 0.0269 & 0.4220 \\
\hline Ruminococcus albus & $0.0114^{\mathrm{b}}$ & $1.7266^{\mathrm{a}}$ & $0.5975^{\mathrm{b}}$ & 0.1804 & 0.0015 \\
\hline Streptococcus bovis & $0.0034^{b}$ & $0.2918^{\mathrm{a}}$ & $0.2086^{\mathrm{a}}$ & 0.0435 & 0.0086 \\
\hline
\end{tabular}

${ }^{1}$ CON Control $(n=4)$, EML Ensiled mulberry leaves $(n=4)$, SMFP sun-dried mulberry fruit pomace $(n=4)$

${ }^{2}$ SEM Standard error of the mean

Population sizes are expressed as percentages of the 165 rRNA gene copy number of the total bacteria

Different letters in the same row indicate a significant difference between values within the row $(p<0.05)$

found to be significantly increased in the EML and SMFP groups, while the Selenomonas ruminantium $(p=0.0591)$, Succinivibrio dextrinosolvens $(p=0.0652)$, and Butyrivibrio fibrisolvens $(p=0.0702)$ populations were increased in these two groups, although the difference in this case was not statistically significant. The
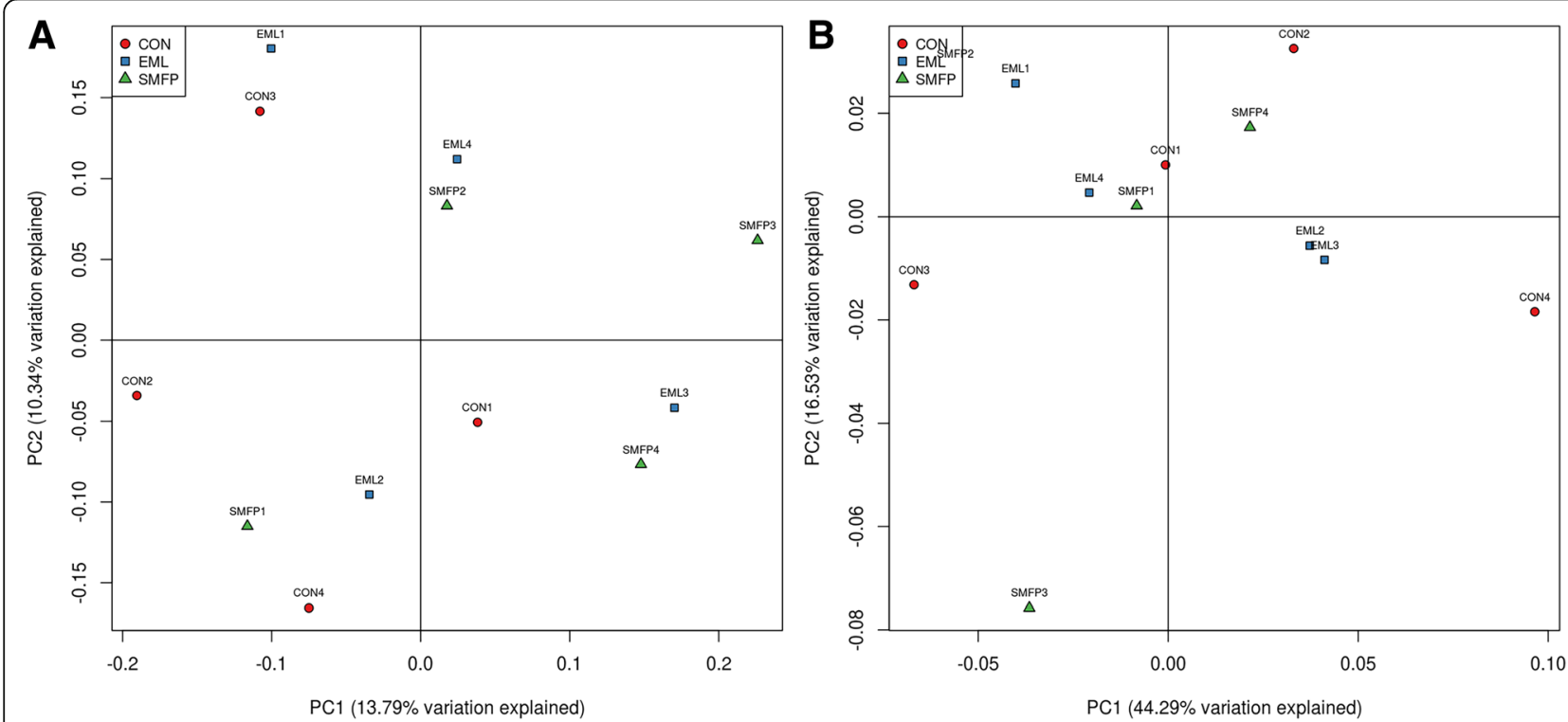

Fig. 4 Weighted (a) and unweighted (b) principal coordinate analysis (PCOA) illustrating relationships among fecal bacterial populations in steers fed different diets 
remaining eight examined bacterial targets showed no difference between the different treatment groups.

\section{Discussion}

The gut bacterial composition of animals is influenced by many factors, and their primary function is to metabolize undigested carbohydrates absorbed by the upper gut, resulting in the production of organic acids, gases, and short-chain fatty acids [20-22]. The bacterial composition of the intestine has a significant impact on the growth and health of cattle and is associated with fecal contamination of environmental sources and human illness via foodborne pathogens [23-26]. Many DNA sequencing studies that have analyzed the feces of beef and dairy cattle from a variety of geographical locations and different management practices have identified a core set of three phyla across all cattle. These three phyla, in order of relative abundance are Firmicutes, Bacteroidetes, and Proteobacteria [27]. This report is in agreement with the dominant phyla reported in many other studies of mammalian gut microbiota [28-30] as well as in our present study. Firmicutes and Bacteroidetes were dominant phyla in all fecal samples. The ratio of Firmicutes to Bacteroidetes has been shown to affect energy harvesting and body fat in humans and mice, and a decreased amount of Bacteroidetes in the microbiota was correlated with increased fat in blood and tissue [31, 32]. Research has demonstrated that there is a relationship between dietary efficiency and the diversity of gastrointestinal bacterial populations. Studies on mice have shown that genetically obese mice had a greater population of bacteria from Firmicutes than Bacteroidetes when compared with genetically lean mice $[31,33]$. In the present study, we found no statistically significant differences in the Firmicutes:Bacteroidetes ratio in the fecal population, but we did observe a trend for smaller Firmicutes:Bacteroidetes ratios in the SMFP group. Compared to our previous study [11] involving the analysis of fat content in beef, the fat content of beef was found to be significantly lower in the SMFP group, indicating that certain fatdecreasing components may exist within SMFP products; this result may be due to the altered Firmicutes:Bacteroidetes ratio in this group.

At the genus level, more than $1 \%$ of the total sequences belonged to 5-7 N15, CF231, Oscillospira, Paludibacter, and Akkermansia. This is different from the results of other studies where Prevotella, Ruminococcus, Butyrivibrio, Succiniclasticum, etc., were reported as the dominant genera in the fecal microbial communities in cattle [34-36]. This distinction indicates that the fecal microbial community structure in cattle is greatly affected by diet [37]. Durso et al. indicated that Pervotella was commonly found in cattle feces and associated with dietary differences [38]. Prevotella is believed to play an important role in starch degradation. There are many non-structural carbohydrates and starches present in mulberry fruit pomace [11], which can potentially promote the activity or proliferation of Prevotella. While there was only a slight difference in the population of Prevotella among the three treatment groups, the Prevotella population was slightly increased in the SMFP group compared to the other two groups. Oscillospira seems to be positively correlated with starch content. In humans, Oscillospira was increased in diets containing resistant starch [39]. Cattle fed diets with a high starch content have been shown to have increased bypass of starch from the rumen [40] and Oscillospira in cattle feces may be associated with the high levels of starch bypass. Ruminal Oscillospira are positively correlated with diets rich in forage [41]. While there were no significant differences in the bacterial abundance between the three groups in our result, the addition of supplement at low level may be responsible for the lack of change in fecal bacterial abundance of Oscillospira.

Many previous studies investigating the microbial community in the digestive system of ruminants have focused on the rumen, which is the largest and most important compartment of the stomach in ruminants [42-45]. However, as the large intestine, especially the caecum and colon, is an important organ for nutrition supply and absorption in ruminants, it has received considerable research attention [46]. Some studies have suggested that the large intestine provides an active fermentation condition similar to the reticulum-rumen. Through quantitative PCR, we were able to illustrate changes in individual target species, and these species are among the most commonly researched fecal microbial species. We recognized Fibrobacter succinogenes, Ruminococcus flavefaciens, and $R$. albus as the major cellulolytic bacterial species, while $S$. ruminantium is the dominant hemicellulosedegrading bacteria [47], which is highly oriented towards fiber degradation. All of the above species are important in the intestine environment, as they are responsible for the digestion of cellulose and hemicellulose in the intestine of ruminants. Cellulose and hemicellulose are mainly digested in the large intestine of cattle, accounting for $18-27 \%$ and $20-40 \%$ of the total digested cellulose and hemicellulose, respectively [48]. It is reasonable that the large intestine has a strong ability to digest cellulose and hemicellulose, as fecal bacteria in the large intestine produce special cellulolytic enzymes that could hydrolyze pentosans and hemicellulose. In this report, the population of $R$. albus was significantly increased in the EML group ( $p=0.0015$ ), but the other cellulolytic bacteria were not influenced by the EML and SMFP diets. This finding indicates that supplementing the diet with EML may positively influence cellulose degradation. Amylolytic species 
of fecal bacteria, including Prevotella bryantii, Prevotella ruminicola, S. bovis, and Ruminobacter amylophilus in the present study, use intracellular amylase to hydrolyze starch to glucose, maltose, maltotriose, and maltotetraose [49]. Our result showed that the abundance of P. bryantii and $P$. ruminicola had an increasing tendency in the EML group, and both the EML and SMFP diets increased the abundance of $R$. amylophilus. The abundance of $S$. bovis was significantly influenced by both the EML and SMFP supplements. Previous studies indicated that the large intestine of cattle contain a small quantity of starch, and that the large intestine is an important organ for digesting glucose and soluble glucosides [50], indicating that EML and SMFP supplementation can have a positive influence on starch degradation in the upper gut and consequently increase the glucose content of the intestine. In our previous research [11], in vitro gas production at $48 \mathrm{~h}$ was significantly higher in the SMFP treatment than in the CON treatment group, indicating that the SMFP diet may produce more fermentable glucose. Most fecal microorganisms have some proteolytic activity, of which Butyrivibrio fibrisolvenscan be regarded as one of highest proteolytic activity strain. Based on the increasing tendency of Butyrivibrio dextrinosolvens in the EML and SMFP groups relative to the control group, we proposed that EML and SMFP supplementation could promote the utilization of protein resources by gut microorganisms.

\section{Conclusion}

In this study, we compared the community composition of fecal microbiota in cattle fed a standard TMR and in those fed with modified TMR diets in which corn grain and cotton seed meal were partially replaced by EML or SMFP. The results of this study demonstrate that addition of EML and SMFP to the TMR only slightly influence the fecal microbial community structure in finishing steers.

\section{Additional file}

Additional file 1: Figure S1. Rarefaction curve for each sample with a cutoff level of $0.03(n=12)$. Figure S2. Double dendrogram of bacteria present in the rumen of steers fed different diets. Table S1. Ingredients and nutrient composition of the experimental diets. (DOCX $323 \mathrm{~kb}$ )

\section{Abbreviations}

ACE: Abundance-based coverage estimator; CON: Control; CP: Crude protein; EML: Ensiled mulberry leaves; NDF: Neutral detergent fiber; OUTs: Operational taxonomic units; PCoA: Principal coordinate analysis; PCR: Polymerase chain reaction; rt-PCR: Real time - polymerase chain reaction; SEM: Standard error of mean; SMFP: Sun-dried mulberry fruit pomace; TMR: Total mixed ration; WSC: Water soluble carbohydrates

\section{Acknowledgments}

We thank Xiaodong Li and Jinwei Zhao for their technical assistance. We also thank the staffs and students of Beef Cattle Research Center of China Agricultural University for their help with animal care and sampling. We are grateful to the native English speaking scientists of Elixigen Company (Huntington Beach, California) for editing our manuscript.

\section{Funding}

This work was supported by the National Natural Science Foundation of China (31372335), the China Agricultural Research System (CARS-38), the National Natural Science Foundation of China (31,372,335 and 31,672,449), and Special Fund for Agro-scientific Research in the Public Interest (201503134). The funding bodies had no role in the design of the study or collection, analysis, or interpretation of data or in writing the manuscript.

\section{Availability of data and materials}

The data supporting the conclusion of this article are included within the article and 16S rRNA gene sequences supporting the results of this article are available in the GenBank Database (https://www.ncbi.nlm.nih.gov/ genbank/) under accession numbers SRR4299452.

\section{Authors' contributions}

$Y L, Q X M, B Z$, and ZMZ designed the study. YL and BZ participated in the animal experiment and biochemical and molecular experiments, $Y L$ analyzed the data and drafted the manuscript. $Y L, Q X M, B Z$, and $Z M Z$ revised the manuscript. All authors read and approved the final manuscript.

\section{Competing interests}

The authors declare that they have no competing interests.

\section{Consent for publication}

Not applicable.

\section{Ethics approval and consent to participate}

The protocol was approved by the China Agricultural University's Animal Welfare and Ethical Committee (Permit No. DK1008). Experiments were performed in accordance with the Regulations for the Administration of Affairs Concerning Experimental Animals (The State Science and Technology Commission of P. R. China, 1988).

\section{Publisher's Note}

Springer Nature remains neutral with regard to jurisdictional claims in published maps and institutional affiliations.

Received: 30 October 2016 Accepted: 17 April 2017

Published online: 21 April 2017

References

1. Famuyiwa O, Ough CS. Grape pomace: possibilities as animal feed. Am J Enol Viticult. 1982;33(1):44-6.

2. Spanghero M, Salem A, Robinson PH. Chemical composition, including secondary metabolites, and rumen fermentability of seeds and pulp of Californian (USA) and Italian grape pomaces. Anim Feed Sci Tech. 2009;152(3):243-55.

3. Alipour D, Rouzbehan Y. Effects of ensiling grape pomace and addition of polyethylene glycol on in vitro gas production and microbial biomass yield. Anim Feed Sci Tech. 2007:137(1-2):138-49.

4. Pirmohammadi R, Rouzbehan Y, Rezayazdi K, Zahedifar M. Chemical composition, digestibility and in situ degradability of dried and ensiled apple pomace and maize silage. Small Ruminant Res. 2006;66(1):150-5.

5. Alibes X, Munoz F, Rodriguez J. Feeding value of apple pomace silage for sheep. Anim Feed Sci Tech. 1984;11(3):189-97.

6. Fondevila M, Guada JA, Gasa J, Castrillo C. Tomato pomace as a protein supplement for growing lambs. Small Ruminant Res. 1994;13(2):117-26.

7. Lale H, Ozcagiran R. A study on pomological, phenologic and fruit quality characteristics of Mulberry (Morus sp.) species: MSc Thesis, Ege Univ., Izmir, Turkey; 1992.

8. Gerasopoulos D, Stavroulakis G. Quality characteristics of four mulberry (Morus sp) cultivars in the area of Chania, Greece. J Sci Food Agr. 1997:73(2):261-4.

9. Ozdemir F, Topuz A. Some chemical composition of mulberries grown in Antalya. Derim. 1998;15(1):30-5.

10. Elmacı Y, Altuğ T. Flavour evaluation of three black mulberry (Morus nigra) cultivars using GC/MS, chemical and sensory data. J Sci Food Agr. 2002;82(6):632-5 
11. Zhou B, Meng Q, Ren L, Shi F, Wei Z, Zhou Z. Evaluation of chemical composition, in situ degradability and in vitro gas production of ensiled and sun-dried mulberry pomace. J Anim Feed Sci. 2012;21(1):188-197.

12. Zhou Z, Zhou B, Ren L, Meng Q. Effect of ensiled mulberry leaves and sun-dried mulberry fruit Pomace on finishing steer growth performance, blood biochemical parameters, and carcass characteristics. PLoS One. 2014;9(1):e85406.

13. Niu Y, Meng Q, Li S, Ren L, Zhou B, Schonewille T, Zhou Z. Effects of diets supplemented with ensiled mulberry leaves and sun-dried mulberry fruit Pomace on the Ruminal bacterial and Archaeal community composition of finishing steers. PLoS One. 2016;11(6):e0156836.

14. Mao $S$, Zhang R, Wang D, Zhu W. The diversity of the fecal bacterial community and its relationship with the concentration of volatile fatty acids in the feces during subacute rumen acidosis in dairy cows. BMC Vet Res. 2012;8:237.

15. Shanks OC, Kelty CA, Archibeque S, Jenkins M, Newton RJ, McLellan SL, et al. Community structures of fecal bacteria in cattle from different animal feeding operations. Appl Environ Microb. 2011 2011-05-01;77(9):2992-3001.

16. Jeong J, Park $\mathrm{H}$, Lee $\mathrm{K}$, Weon $\mathrm{H}$, Ka J. Microbial community analysis and identification of alternative host-specific fecal indicators in fecal and river water samples using pyrosequencing. J Microbiol. 2011;49(4):585-94.

17. Yu Z, Morrison M. Improved extraction of PCR-quality community DNA from digesta and fecal samples. Bio Techniques. 2004;36(5):808-12.

18. Caporaso JG, Lauber CL, Walters WA, Berg-Lyons D, Huntley J, Fierer N, Owens SM, Betley J, Fraser L, Bauer M, Gormley N, Gilbert JA, Smith G, Knight R. Ultra-high-throughput microbial community analysis on the lllumina HiSeq and MiSeq platforms. ISME J. 2012;6(8):1621-4. doi:10.1038/ismej.2012.8.

19. Caporaso JG, Kuczynski J, Stombaugh J, Bittinger K, Bushman FD, Costello EK, et al. QIIME allows analysis of high-throughput community sequencing data. Nat Methods. 2010;7(5):335-6.

20. Cummings JH, Macfarlane GT. Collaborative JPEN-clinical nutrition scientific publications role of intestinal bacteria in nutrient metabolism. Jpen-Parenter Enter. 1997;21(6):357-65.

21. Lin B, Henderson G, Zou C, Cox F, Liang X, Janssen PH, et al. Characterization of the rumen microbial community composition of buffalo breeds consuming diets typical of dairy production systems in southern China. Anim Feed Sci Tech. 2015;207:75-84.

22. Dowd SE, Callaway TR, Wolcott RD, Sun Y, McKeehan T, Hagevoort RG, et al. Evaluation of the bacterial diversity in the feces of cattle using $16 \mathrm{~S}$ rDNA bacterial tag-encoded FLX amplicon pyrosequencing (bTEFAP). BMC Microbiol. 2008;8(1):125.

23. Abnous K, Brooks SPJ, Kwan J, Matias F, Green-Johnson J, Selinger LB, et al, Diets enriched in oat bran or wheat bran temporally and differentially Alter the composition of the fecal Community of Rats. J Nutr. 2009 2009-11-01; 139(11):2024-2031

24. Hooper LV, Gordon J. Commensal host-bacterial relationships in the gut. Science. 2001;292(5519):1115-8.

25. Cui Z, Meng Q, Ma W, Zhang X, Zhou Z, Ren L. Diversity of the intestinal bacteria of cattle fed on diets with different doses of gelatinized starchurea. Indian J Microbiol. 2015;55(3):269-77.

26. Ellis RJ, Bruce KD, Jenkins C, Stothard JR, Ajarova L, Mugisha L, et al. Comparison of the distal gut Microbiota from People and animals in Africa. PLoS One. 2013 2013-01-23;8(1):e54783.

27. Rice WC, Galyean ML, Cox SB, Dowd SE, Cole NA. Influence of wet distillers grains diets on beef cattle fecal bacterial community structure. BMC Microbiol. [Journal Article]. 2012 2012-01-20;12:25.

28. Arumugam M, Raes J, Pelletier E, Le Paslier D, Yamada T, Mende DR, et al. Enterotypes of the human gut microbiome. Nature. 2011;473(7346):174-80

29. Jami E, Mizrahi I. Composition and similarity of bovine rumen microbiota across individual animals. PLoS One. 2012;7(3):e33306.

30. Ochman H, Worobey M, Kuo C, Ndjango JN, Peeters M, Hahn BH, et al. Evolutionary relationships of wild hominids recapitulated by gut microbial communities. PLoS Biol. 2010;8(11):e1000546.

31. Ley RE, Turnbaugh PJ, Klein S, Gordon JI. Microbial ecology: human gut microbes associated with obesity. Nature. 2006;444(7122):1022-3.

32. Turnbaugh PJ, Ley RE, Mahowald MA, Magrini V, Mardis ER, Gordon Jl. An obesity-associated gut microbiome with increased capacity for energy harvest. Nature. 2006:444(7122):1027-131.

33. Callaway TR, Dowd SE, Edrington TS, Anderson RC, Krueger N, Bauer N, et al. Evaluation of bacterial diversity in the rumen and feces of cattle fed different levels of dried distillers grains plus solubles using bacterial tag-encoded FLX amplicon pyrosequencing. J Anim Sci. 2010 2010-12-01;88(12):3977-3983.

34. Li Y, Hu L, Xue G, Liu H, Yang H, Zhu Y, et al. Comparison of bacterial diversity in large intestine of Xiangxi yellow cattle (Bos taurus) associated with different diet: fresh Miscanthus sinensis and mixed forage. Afr J Microbiol Res. 2012;6:5965-74.

35. Bernhard AE, Field KG. A PCR assay to discriminate human and ruminant feces on the basis of host differences in Bacteroides-Prevotella genes encoding 16S rRNA. Appl Environ Microb. 2000;66(10):4571-4.

36. Sunvold GD, Hussein HS, Fahey GC, Merchen NR, Reinhart GA. In vitro fermentation of cellulose, beet pulp, citrus pulp, and citrus pectin using fecal inoculum from cats, dogs, horses, humans, and pigs and ruminal fluid from cattle. J Anim Sci. 1995;73(12):3639-48.

37. Kim M, Kim J, Kuehn LA, Bono JL, Berry ED, Kalchayanand N, et al. Investigation of bacterial diversity in the feces of cattle fed different diets. J Anim Sci. 2014;92(2):683-94

38. Durso LM, Wells JE, Harhay GP, Rice WC, Kuehn L, Bono JL, et al. Comparison of bacterial communities in faeces of beef cattle fed diets containing corn and wet distillers' grain with solubles. Lett Appl Microbiol. 2012:55(2):109-14.

39. Walker AW, Ince J, Duncan SH, Webster LM, Holtrop G, Ze X, et al. Dominant and diet-responsive groups of bacteria within the human colonic microbiota. ISME J. 2011:5(2):220-30.

40. Wells JE, Shackelford SD, Berry ED, Kalchayanand N, Guerini MN, Varel VH, et al. Prevalence and level of Escherichia coli 0157: H7 in feces and on hides of feedlot steers fed diets with or without wet distillers grains with solubles. J Food Prot. 2009:72(8):1624-33.

41. Mackie Rl, Aminov Rl, Hu W, Klieve AV, Ouwerkerk D, Sundset MA, et al. Ecology of uncultivated Oscillospira species in the rumen of cattle, sheep, and reindeer as assessed by microscopy and molecular approaches. Appl Environ Microb. 2003;69(11):6808-15.

42. Tajima K, Arai S, Ogata K, Nagamine T, Matsui H, Nakamura M, et al. Rumen bacterial community transition during adaptation to high-grain diet. Anaerobe. 2000;6(5):273-84

43. Mao SY, Zhang RY, Wang DS, Zhu WY. Impact of subacute ruminal acidosis (SARA) adaptation on rumen microbiota in dairy cattle using pyrosequencing. Anaerobe. 2013;24:12-9.

44. Rius AG, Kittelmann S, Macdonald KA, Waghorn GC, Janssen PH, Sikkema E. Nitrogen metabolism and rumen microbial enumeration in lactating cows with divergent residual feed intake fed high-digestibility pasture. J Dairy Sci. 2012;95(9):5024-34.

45. De Barbieri I, Hegarty RS, Silveira C, Gulino LM, Oddy VH, Gilbert RA, et al. Programming rumen bacterial communities in newborn merino lambs. Small Ruminant Res. 2015;129:48-59.

46. Pitta DW, Pinchak WE, Dowd SE, Osterstock J, Gontcharova V, Youn E, et al. Rumen bacterial diversity dynamics associated with changing from bermudagrass hay to grazed winter wheat diets. Microbial Ecol. 2010;59(3):511-22.

47. Flint $\mathrm{HJ}$, Bayer EA, Rincon MT, Lamed $\mathrm{R}$, White BA. Polysaccharide utilization by gut bacteria: potential for new insights from genomic analysis. Nat Rev Microbiol. 2008:6(2):121-31.

48. Warner RL, Mitchell GE, Little CO. Post-ruminal digestion of cellulose in wethers and steers. J Anim Sci. 1972:34(1):161-5.

49. Cotta MA. Interaction of ruminal bacteria in the production and utilization of maltooligosaccharides from starch. Appl Environ Microb. 1992;58(1):48-54.

50. Noziere P, Rémond D, Lemosquet S, Chauveau B, Durand D, Poncet C. Effect of site of starch digestion on portal nutrient net fluxes in steers. Brit J Nutr. 2005:94(02):182-91. 